\title{
Reduced CD4 type I cytokine response by HIV-I transgenic rat may be correlated with increased SOCS-I expression from dendritic
} \section{cells}

\author{
Anjana Yadav and William C Reid*
}

Address: Division of Basic Science, Institute of Human Virology, University of Maryland Biotechnology Institute, Baltimore, Maryland, 21201, USA

* Corresponding author

from 2006 International Meeting of The Institute of Human Virology

Baltimore, USA. |7-2I November, 2006

Published: 21 December 2006

Retrovirology 2006, 3(SuppI I):SI03 doi:I0.II86/I742-4690-3-SI-SI03

(c) 2006 Yadav and Reid; licensee BioMed Central Ltd.

Suppressor of cytokine signaling-1 (SOCS-1) is an inducible negative regulator of the JAK/STAT signal pathway; SOCS-1 is expressed in dendritic cells (DCs) and negatively regulates activation and type 1 regulatory cytokine production. Infection with human immunodeficiency virus type 1 (HIV-1) results in cytokine dysregulation by DCs. Since effector/memory formation depends upon clonal expansion of naïve $\mathrm{T}$ cells following the initial encounter with antigen and since cytokines play a critical role in the initiation and regulation of immune responses by DCs, events that negatively affect antigen presentation, co-stimulation and cytokine production by DCs can also negatively affect $\mathrm{T}$ cell immune responses. We have earlier reported that HIV-1 transgenic rats have defects in type 1 cytokine production, type 1 cytokine responses and generation of effector/memory CD4 T cell subsets. Here we show that BMDC from HIV-1 transgenic rats express significantly elevated levels of IL10 and reduced levels of IL12 proteins in addition to elevated SOCS-1 mRNA following stimulation with lipolysaccharide (LPS) compared to age matched controls. These results suggest that elevated levels of SOCS-1 in DCs may negatively regulate type 1 cytokine production leading to dysregulation of $\mathrm{T}$ helper 1 responses reported in the HIV-1 transgenic rat.

\section{Acknowledgements}

NIH-NIAID ROI-AI63I7I. 